\title{
Graphene-passivated nickel as an efficient hole-injecting electrode for large area organic semiconductor devices
}

Cite as: Appl. Phys. Lett. 116, 163301 (2020); https://doi.org/10.1063/5.0002222

Submitted: 23 January 2020 . Accepted: 29 March 2020 . Published Online: 20 April 2020

Daniele Di Nuzzo (D), Ryo Mizuta (D), Kenichi Nakanishi, Marie-Blandine Martin, Adrianus I. Aria (D), Robert Weatherup (iD, Richard H. Friend, Stephan Hofmann (D), and Jack Alexander-Webber (D)

\section{ARTICLES YOU MAY BE INTERESTED IN}

Direct observation of charge accumulation in quantum well solar cells by cross-sectional Kelvin probe force microscopy

Applied Physics Letters 116, 163501 (2020); https://doi.org/10.1063/1.5142438

Scaling magnetic tunnel junction down to single-digit nanometers-Challenges and prospects Applied Physics Letters 116, 160501 (2020); https://doi.org/10.1063/5.0004434

On-chip terahertz modulation and emission with integrated graphene junctions

Applied Physics Letters 116, 161104 (2020); https://doi.org/10.1063/5.0005870

\section{Lock-in Amplifiers up to $600 \mathrm{MHz}$}
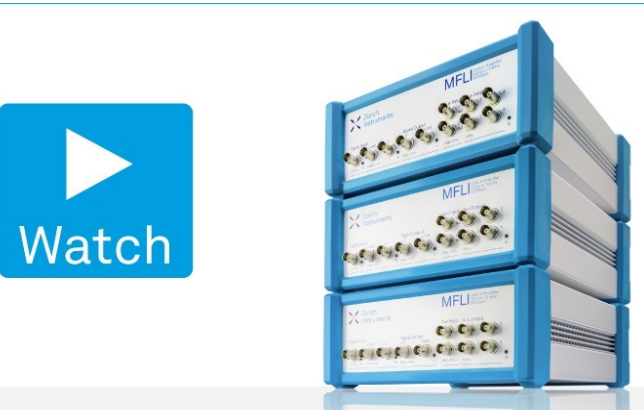


\title{
Graphene-passivated nickel as an efficient hole-injecting electrode for large area organic semiconductor devices
}

Cite as: Appl. Phys. Lett. 116, 163301 (2020); doi: 10.1063/5.0002222

Submitted: 23 January 2020 - Accepted: 29 March 2020 •

Published Online: 20 April 2020

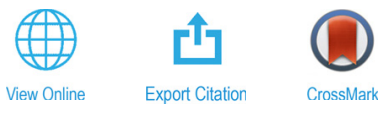

Daniele Di Nuzzo, ${ }^{1, a)}$ (D) Ryo Mizuta, ${ }^{2}$ (D Kenichi Nakanishi, ${ }^{2}$ Marie-Blandine Martin, ${ }^{3}$ Adrianus I. Aria, ${ }^{4}$ Robert Weatherup, ${ }^{5}$ (iD Richard H. Friend, ${ }^{7}$ Stephan Hofmann, ${ }^{2}$ (D) and Jack Alexander-Webber ${ }^{2, a)}$ (iD)

\begin{abstract}
AFFILIATIONS
${ }^{7}$ Cavendish Laboratory, University of Cambridge, Cambridge CB3 OHE, United Kingdom

${ }^{2}$ Department of Engineering, University of Cambridge, Cambridge CB3 OFA, United Kingdom

${ }^{3}$ Unité Mixte de Physique, CNRS, Thales, Université Paris-Saclay, 91767, Palaiseau, France

${ }^{4}$ Surface Engineering and Precision Institute, School of Aerospace, Transport and Manufacturing, Cranfield University, Cranfield MK43 OAL, United Kingdom

${ }^{5}$ Department of Materials, University of Oxford, Parks Road, Oxford OX1 3PH, United Kingdom
\end{abstract}

${ }^{a)}$ Authors to whom correspondence should be addressed: dd467@cantab.ac.uk and jaa59@cam.ac.uk

\begin{abstract}
Efficient injection of charge from metal electrodes into semiconductors is of paramount importance to obtain high performance optoelectronic devices. The quality of the interface between the electrode and the semiconductor must, therefore, be carefully controlled. The case of organic semiconductors presents specific problems: ambient deposition techniques, such as solution processing, restrict the choice of electrodes to those not prone to oxidation, limiting potential applications. Additionally, damage to the semiconductor in sputter coating or high temperature thermal evaporation poses an obstacle to the use of many device-relevant metals as top electrodes in vertical metal-semiconductor-metal structures, making it preferable to use them as bottom electrodes. Here, we propose a possible solution to these problems by implementing graphene-passivated nickel as an air stable bottom electrode in vertical devices comprising organic semiconductors. We use these passivated layers as hole-injecting bottom electrodes, and we show that efficient charge injection can be achieved into standard organic semiconducting polymers, owing to an oxide free nickel/graphene/polymer interface. Crucially, we fabricate our electrodes with low roughness, which, in turn, allows us to produce large area devices (of the order of millimeter squares) without electrical shorts occurring. Our results make these graphene-passivated ferromagnetic electrodes a promising approach for large area organic optoelectronic and spintronic devices.
\end{abstract}

Published under license by AIP Publishing. https://doi.org/10.1063/5.0002222

Organic semiconductors serve as a platform for (opto)electronic devices with tunable characteristics by molecular design, enabling versatile device integration, and processing strategies. ${ }^{1}$ However, ambient processing techniques such as solution processing can facilitate oxidation of metal contacts, resulting in an uncontrolled electronic interface, which is deleterious to performance in semiconductor devices. ${ }^{2,3} \mathrm{New}$ techniques are, therefore, required to control the interface between organic semiconductors and oxidizing metals while maintaining the possibility of solution processing.

Graphene has been shown to act as an atomically thin permeation barrier. ${ }^{4-6}$ Graphene grown via chemical vapor deposition (CVD) directly on the surface of strongly interacting ${ }^{7}$ catalytic metals, such as $\mathrm{Ni}, \mathrm{Co}$, or $\mathrm{Fe}$, acts as a barrier layer to prevent oxidation. ${ }^{8-11}$ These oxide-free ferromagnetic interfaces have been shown to hold significant benefits within the field of spintronics, ${ }^{12,13}$ as they enable oxidative fabrication processes, such as solution processing ${ }^{9,10}$ or atomic layer deposition, ${ }^{14}$ to be used to fabricate devices with a wider range of relevant materials. One appealing possibility would be to develop graphene-passivated ferromagnets as electrodes ${ }^{9,10}$ for organic semiconductor spintronics, ${ }^{15-18}$ where the quality of the electronic interface between the ferromagnetic electrode and the organic semiconductor is of paramount importance. ${ }^{19,20}$ Another important advantage of an ambient-stable ferromagnetic layer is that it can be used as a bottom electrode in vertical metal-organic semiconductor- metal 
structures, allowing one to employ techniques such as sputtering to obtain high quality and thickness-controlled metal layers or multilayers; note that sputtering cannot be used for top-electrodes as it would destroy ${ }^{21}$ the organic semiconductor.

Previous reports using graphene passivated ferromagnets as electrodes for organic semiconductor devices have studied the spin injection properties ${ }^{10}$ as well as charge injection in lateral organic semiconductor field effect transistors. ${ }^{9}$ In this work, we investigate few-layer graphene-passivated nickel (Ni/FLG) as a bottom electrode for injection of holes into organic semiconducting polymers in a vertical device structure, demonstrating efficient injection into two standard semiconducting polymers deposited from solution and in air, directly on top of $\mathrm{Ni}$ / FLG. Compared to previous reports on graphene-passivated ferromagnetic electrodes, where lithographic techniques had to be used in order to produce micrometer-sized features, ${ }^{8-10,12,14}$ here, we were able to produce working devices with several orders of magnitude larger active area $\left(4.5 \mathrm{~mm}^{2}\right)$. Our results are, thus, encouraging for the further development of organic optoelectronic and spintronic devices processed from solution under ambient conditions.

Nickel was initially sputtered on thermally oxidized silicon wafers, producing films with a thickness of $150 \mathrm{~nm}$. FLG domains were grown on such sputtered $\mathrm{Ni}$ films in a custom low-pressure Chemical Vapor Deposition (CVD) reactor (base pressure $\sim 1$ $\times 10^{-6} \mathrm{mbar}$ ). All substrates were cleaned by sonicating in acetone followed by isopropyl alcohol and blow-dried with a nitrogen gun before loading. Samples were heated to approximately $450{ }^{\circ} \mathrm{C}$ using a resistive heater (temperature measurements by a K-type thermocouple) with a rapid ramp rate of $100^{\circ} \mathrm{C} / \mathrm{min}$ and annealed at $\sim 1 \mathrm{mbar}$ of $\mathrm{H}_{2}$ for $10 \mathrm{~min}$. This reduces the native oxide prior to graphene growth. After annealing, the $\mathrm{H}_{2}$ flow was stopped and the chamber was evacuated back to approximately base pressure over a period of $5 \mathrm{~min}$. For graphene growth, $\mathrm{C}_{2} \mathrm{H}_{2}$ gas was gradually introduced into the reactor via a mass flow controller by incrementally increasing the flow rate over $5 \mathrm{~min}$ to achieve a partial pressure of $\mathrm{C}_{2} \mathrm{H}_{2}$ of $2.5 \times 10^{-4}$ mbar. Subsequently, the samples were held at $450^{\circ} \mathrm{C}$ in $2.5 \times 10^{-4} \mathrm{mbar}$ of $\mathrm{C}_{2} \mathrm{H}_{2}$ for a further $25 \mathrm{~min}$, before rapid cooling (initially $\sim 300^{\circ} \mathrm{C} / \mathrm{min}$ ) while maintaining the $\mathrm{C}_{2} \mathrm{H}_{2}$ flow. All gases were stopped once room temperature had been reached.

Upon graphene growth, a roughening of the $\mathrm{Ni}$ sputtered on thermally oxidized $\mathrm{Si}$ was observed, with an RMS $=67 \mathrm{~nm}$ [Fig. 1(a)]. The roughness was found to increase with increasing growth temperature. The roughening of $\mathrm{Ni}$ upon graphene growth is explained by grain growth in the sputtered Ni films, occurring at high temperatures during the CVD process: under these conditions, the internal forces in the film are larger than those between the film and the substrate, and diffusion of the film material is appreciable.

Surface roughness is a significant problem in our desired vertical metal-semiconductor-metal devices: to obtain the cleanest interface and achieve efficient charge injection, the organic semiconductors are spin coated from solution directly on top of the Ni-graphene film without the use of roughness-reducing intermediate layers; however, if the surface of the $\mathrm{Ni}$-graphene film is too rough, $\mathrm{Ni}$ protrudes through the film and can directly come into contact with the top electrode, causing electrical shorting of the devices. While a thick layer of organic semiconductor may be used to contain this problem, the operating voltage of the devices will scale with the semiconductor thickness, resulting in impractical voltages being required to operate the devices.
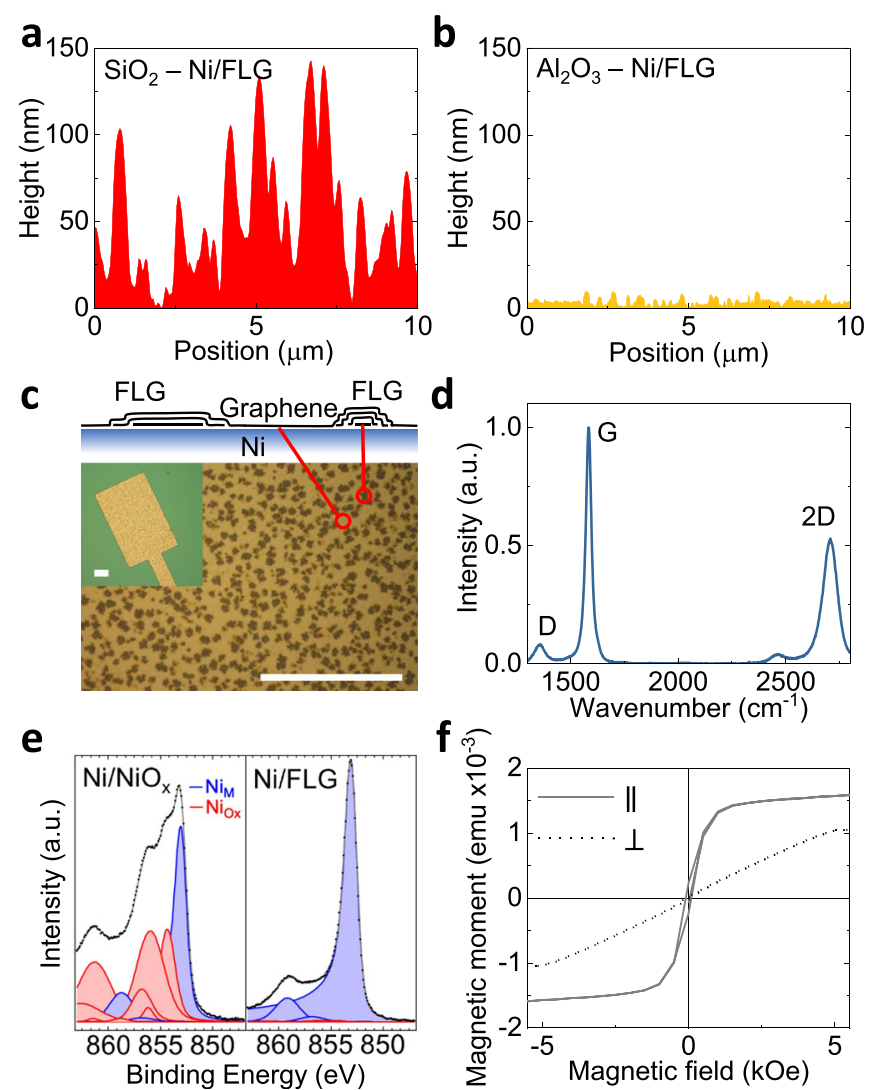

FIG. 1. AFM line scan of sputtered Ni films after graphene growth using a $\mathrm{Si}-\mathrm{SiO}_{2}$ substrate without (a) and with (b) an $\mathrm{Al}_{2} \mathrm{O}_{3}$ adhesion layer. (c) Optical micrograph of a graphene-passivated Ni electrode, including a cartoon indicating areas with different numbers of graphene layers. Scale bars are $50 \mu \mathrm{m}$. (d) Raman spectrum of few-layer graphene on $\mathrm{Ni}$. (e) XPS of the $\mathrm{Ni} 2 \mathrm{p}_{3 / 2}$ region of sputtered $\mathrm{Ni}\left(\mathrm{Ni} / \mathrm{NiO}_{x}-\right.$ black) and $\mathrm{Ni}$ after multilayer graphene growth (Ni/FLG-green), with peak fitting indicating metallic nickel $\left(\mathrm{Ni}_{\mathrm{M}}\right)$ and nickel oxide $\left(\mathrm{Ni}_{\mathrm{Ox}}\right)$. (f) Magnetic moment measured at room temperature by DC-mode SQUID magnetometry of graphenepassivated $\mathrm{Ni}$ films for magnetic fields parallel (gray, solid) and perpendicular (black, dots) to the plane of the film.

To solve the issue of roughness, we reduced grain growth by adding a $10 \mathrm{~nm} \mathrm{Al}_{2} \mathrm{O}_{3}$ adhesion-promoting layer, ${ }^{22}$ grown by atomic layer deposition on the $\mathrm{Si}$ wafer prior to $\mathrm{Ni}$ deposition. The $\mathrm{Al}_{2} \mathrm{O}_{3}$ adhesion-promoting layer sensibly reduced the surface roughness [Fig. $1(\mathrm{~b}), \mathrm{RMS}=4.3 \mathrm{~nm}$ ]: as presented below, this allowed us to reliably fabricate working devices showing no electrical shorts and with a surface area of $4.5 \mathrm{~mm}^{2}$, which is large compared to commonly studied vertical spintronic devices (typically on a scale between 1 and $\left.10^{4} \mu \mathrm{m}^{2}\right)^{8-10,12,14}$

Optical microscopy [Fig. 1(c)] and Raman spectroscopy [Fig. 1(d)] confirmed that the electrodes were fully covered with graphene, notably with islands of multiple layers, as is often observed under similar growth conditions on Ni thin films. ${ }^{11,23,24}$ Such thicker multilayer islands appeared as dark sections in optical microscopy, as shown schematically in Fig. 1(c). Raman spectroscopy was carried out using a Renishaw in Via confocal Raman microscope and a laser excitation wavelength of $532 \mathrm{~nm}$. We observed a D peak [Fig. 1(d)], which we 
ascribe to the relatively low temperature of the growth process. The broad $2 \mathrm{D}$ peak $\left(\mathrm{FWHM}=79 \mathrm{~cm}^{-1}\right)$ and $\mathrm{G} / 2 \mathrm{D}$ ratio $>1$ are also consistent with multilayer growth. ${ }^{25}$

X-ray photoelectron spectroscopy (XPS) was carried out using a Thermo Scientific ESCALAB 250Xi with a micro-focused and monochromated $\mathrm{Al} \mathrm{K \alpha}$ x-ray source. All spectra were background-corrected (Shirley) and analyzed by performing a nonlinear mean square fit of the data, using Doniach-Sùnjić functions convoluted with Gaussian profiles. Measurements of the $\mathrm{Ni}_{2} \mathrm{p}_{3 / 2}$ region confirmed that the sputtered $\mathrm{Ni}$ is initially oxidized during air transfer, with significant nickel oxide/hydroxide $\left(\mathrm{Ni}_{\mathrm{Ox}}\right)$ components visible [Fig. 1(e), left]. Following graphene growth, the previously oxidized $\mathrm{Ni}$ is reduced to metallic $\mathrm{Ni}$ and remains reduced despite transfer of the electrodes through air, as confirmed by the dominant $\mathrm{Ni}_{\mathrm{M}}$ components and negligible contributions from $\mathrm{Ni}_{\mathrm{Ox}}$ species [Fig. $1(\mathrm{e})$, right]. This effective passivation is attributable to the strong interaction between graphene and $\mathrm{Ni}$ and the fact that even if defects are present in the graphene, a passivating oxide is formed locally, which does not propagate laterally. ${ }^{26}$ Herein, we used an extended hydrocarbon exposure with the intention of blocking as many defects as possible through the formation of thicker FLG at these locations, where hydrocarbon can continue to access the Ni surface [Fig. 1(c)].

We used a Quantum Design SQUID magnetometer (MPMS3 model) to determine the room temperature magnetic properties of the Ni films after graphene growth [Fig. 1(f)]. With a standard DC-mode measurement, we determined the magnetization of our films parallel and perpendicular to the magnetic field: consistent with previous observations, ${ }^{27}$ the easy-axis of magnetization of the Ni films is parallel to the plane of the film. The films also show the typical small hysteresis of nickel, that is of a "soft" ferromagnetic metal. These measurements, thus, confirm that the bulk ferromagnetic properties of the Ni layers are maintained after graphene passivation.

We then fabricated devices with the structure schematically depicted in Fig. 2(a). Electrodes consisting of $150 \mathrm{~nm}$ thick stripes of $\mathrm{Ni}$ were patterned by using shadow masks on a $\mathrm{Si}$ wafer with an $\mathrm{Al}_{2} \mathrm{O}_{3}$ adhesion layer; few-layer graphene was grown on top of $\mathrm{Ni}$ as described above. We first studied hole injection into the organic semiconducting polymer F8BT (poly(9,9-dioctylfluorene-alt-benzothiadiazole)), a polymer commonly used in green emitting organic light emitting diodes (OLEDs). F8BT was previously received from Cambridge Display Technology, and it was spin coated in air directly on top of the bottom electrode. A solution of $15 \mathrm{mg} / \mathrm{ml}$ in para-xylene was used for spin-coating, giving smooth and homogeneous films with a thickness of $180 \mathrm{~nm}$ (measured using a Dektak profilometer). A top electrode consisting of $\mathrm{MoO}_{3} / \mathrm{Au}(8 \mathrm{~nm} / 100 \mathrm{~nm})$ was then deposited by thermal evaporation at a pressure of $10^{-7} \mathrm{mbar}$.

The device area, determined by the overlap of the top and bottom contacts, was $4.5 \mathrm{~mm}^{2}$. The $\mathrm{MoO}_{3} / \mathrm{Au}$ contact was used to study Ohmic injection into the deep-lying HOMO level of F8BT $(-5.9 \mathrm{eV}) .{ }^{28}$ Ohmic injection of holes has been demonstrated previously using $\mathrm{MoO}_{3}$ and is due to surface p-doping of the polymer by $\mathrm{MoO}_{3}$ at the polymer interface, ${ }^{29}$ which results in barrier-free injection. $^{30}$ Figure 2 (b) shows the current density-voltage characteristic measured on the devices. Negative voltages correspond to injection of holes from $\mathrm{MoO}_{3} / \mathrm{Au}$ : Ohmic injection is achieved as expected, evidenced by high currents reaching the space-charge limit at high absolute voltages.
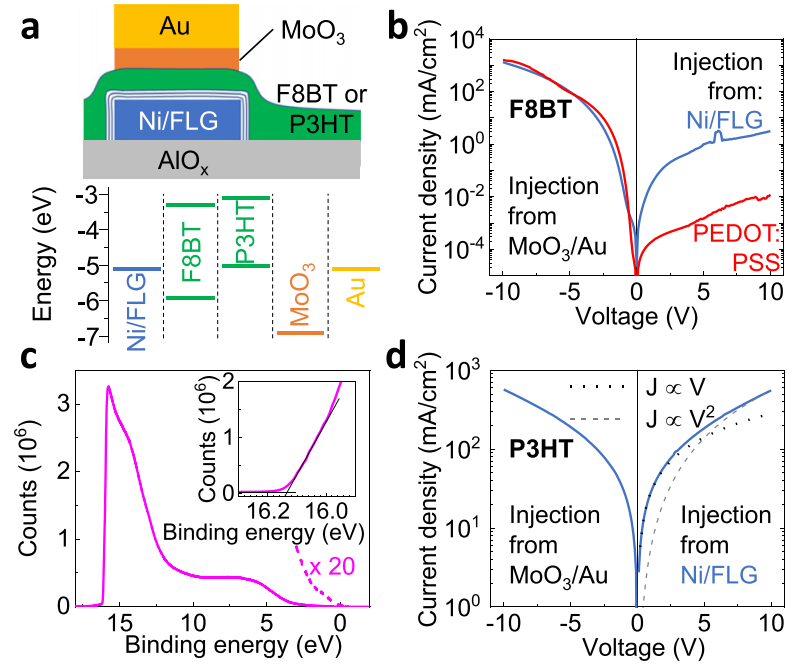

FIG. 2. (a) Schematic representation of the device structure used. (b) Comparison of hole injection into F8BT from graphene-passivated Ni (blue) and PEDOT:PSS (red) bottom contacts, each using a top contact of $\mathrm{MoO}_{3} / \mathrm{Au}$. (c) Binding energy, referenced to the Fermi level, measured on a Ni/FLG sample by means of UPS (excitation light with energy $\mathrm{h} \nu=21.21 \mathrm{eV}$ ). The inset shows the high binding energy part of the spectrum used to extract the cutoff energy $E_{\mathrm{co}}$. The work function $\phi$ is calculated as $\phi=h \nu-E_{c o}$. For better visualization, the part of the curve at the Fermi level is also shown after 20-fold magnification (dashed line). (d) Hole injection into $\mathrm{P} 3 \mathrm{HT}$ from graphene-passivated $\mathrm{Ni}$, with the $\mathrm{MoO}_{3} / \mathrm{Au}$ top contact.

The Ni/FLG devices were compared against reference devices that have the same surface area and consist of the standard structure ITO/PEDOT:PSS/F8BT/MoO $/$ /Au. For PEDOT:PSS, the Heraeus Clevios ${ }^{\text {TM }}$ AI 4083 formulation was used. The PEDOT:PSS film was deposited in air on top of a cleaned, pre-patterned ITO substrate and subsequently annealed under nitrogen for $20 \mathrm{~min}$ at $150^{\circ} \mathrm{C}$. The remaining part of the vertical stack was fabricated as in the case of Ni/FLG-based devices.

When injecting from $\mathrm{MoO}_{3} / \mathrm{Au}$, the same behavior as in the $\mathrm{Ni} /$ FLG devices was observed. When injecting from PEDOT:PSS into F8BT, current densities ca. five orders of magnitude lower were observed, consistent with injection of holes being barrier-limited. While we still observed a barrier-limited injection, when injecting holes from Ni/FLG, we recorded current densities that were $2-3$ orders of magnitude higher than that in the case of PEDOT:PSS.

We further characterized the Ni/FLG layers to obtain more information about their electrical properties in devices. We measured the resistivity of the Ni/FLG films using the four-point probe method and recorded a resistivity of $1.5 \times 10^{-7} \Omega \mathrm{m}$. This translates in a series resistance of the whole $\mathrm{Ni} / \mathrm{FLG}$ lead of $\sim 1 \Omega$, therefore representing a negligible contribution to the total resistance of the device.

We then measured the work function of our Ni/FLG substrates by means of ultraviolet photoelectron spectroscopy (UPS) using a Thermo Fisher Scientific ESCALAB 250Xi x-ray/Ultraviolet (XPS/ UPS) photoelectron spectrometer, with an excitation wavelength of $21.21 \mathrm{eV}$; consistent with previous reports, our Ni/FLG samples show a work function of $5.07 \mathrm{eV}$ [Fig. 2(c)], which is close to the work function of nickel. ${ }^{31-33}$ A substantial energy barrier is, therefore, present at the Ni/FLG/F8BT interface, resulting in the barrier-limited injection 
observed in Fig. 2(b). Note that the measured work function of $\mathrm{Ni} / \mathrm{FLG}$ is comparable to the work function of the same PEDOT:PSS formulation, previously reported to be $5.1 \mathrm{eV} .{ }^{34}$ However, charge accumulation at the Ni/FLG/F8BT interface under voltage bias could shift the work function downwards, as it has been previously shown in graphene; ${ }^{35,36}$ we hypothesize that this effect might contribute to a lower effective injection barrier at the interface with Ni/FLG compared to PEDOT:PSS, thus explaining the difference in current density observed at positive bias in F8BT-based devices [Fig. 2(b)].

We tested the same Ni/FLG/polymer/ $/ \mathrm{MoO}_{3} / \mathrm{Au}$ structure using another standard semiconducting polymer: P3HT (Poly(3-hexylthiophene-2,5-diyl)). P3HT was purchased from Rieke Metals in its regioregular version (catalogue number RMI-00EE) and dissolved in a chlorobenzene:chloroform mixture (50:50, volume:volume) using a concentration of $30 \mathrm{mg} / \mathrm{ml}$; spin-coating in air yielded smooth and homogeneous films of $200 \mathrm{~nm}$ thickness (measured using a Dektak profilometer). $\mathrm{A} \mathrm{MoO}_{3} / \mathrm{Au}(8 / 100 \mathrm{~nm})$ top electrode was evaporated on top of the polymer layer, as in the case of the previously discussed F8BT devices.

In this case, we observed high currents both when injecting from $\mathrm{MoO}_{3} / \mathrm{Au}$ and when injecting from Ni/FLG [Fig. 2(d)], with a very symmetric current density-voltage curve, linear at low voltages and reaching the space-charge current limit at higher voltages (as testified by a $\mathrm{V}^{2}$ dependence of the current density). The perfectly symmetric $\mathrm{J}-\mathrm{V}$ plot demonstrates that hole injection in $\mathrm{P} 3 \mathrm{HT}$ is barrier-free from both contacts. This result is consistent with the absence of an energy barrier between the HOMO level of P3HT $(5.0 \mathrm{eV})$ and Ni/FLG $(5.07 \mathrm{eV}$ as measured in UPS).

To further investigate the electronic quality of nickel passivated electrodes, we compared the hole injection into F8BT from passivated nickel with the case of non-protected nickel electrodes (Fig. 3). Here, we tested smaller scale devices with an active area of $0.05 \mathrm{~mm}^{2}$, fabricated by employing a resist mask patterned by photolithography for the deposition of Ni followed by lift-off in acetone. The applied voltage was set up to probe hole injection from $\mathrm{Ni} / \mathrm{NiO}_{\mathrm{x}}$ into F8BT. We observed that hole injection from unpassivated $\mathrm{Ni} / \mathrm{NiO}_{\mathrm{x}}$ has a very low efficiency [Fig. 3(a)], indicated by low and unstable current densities. A significant improvement is observed with graphene passivation [Fig. 3(b)], suggesting that the native oxide of Ni has a detrimental effect on charge injection.

Furthermore, in Fig. 3, the voltage is swept first from $0 \mathrm{~V}$ to $10 \mathrm{~V}$ and then $10 \mathrm{~V}$ to $0 \mathrm{~V}$ at a sweep rate of $2 \mathrm{~V} \mathrm{~s}^{-1}$ so that any hysteretic effects can be studied. Our $\mathrm{Ni} / \mathrm{NiO}_{\mathrm{x}}$ devices show not only strong instabilities in current but also large hysteretic behavior. We explain
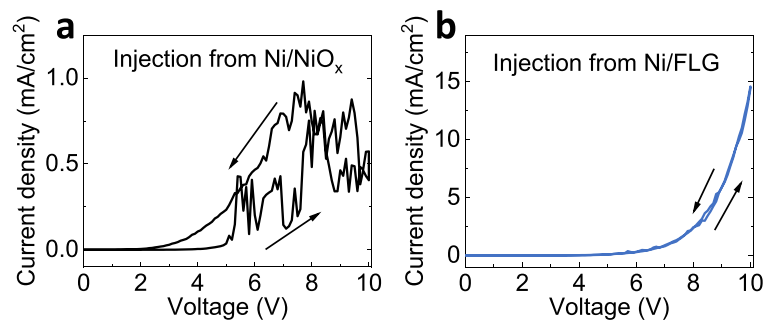

FIG. 3. Current density hysteresis as a function of voltage for hole injection into F8BT using (a) unprotected sputtered Ni electrodes and (b) graphene-passivated Ni. The arrows indicate the direction of voltage sweep. the observed unstable and hysteretic currents as originating from resistive switching processes taking place in $\mathrm{NiO}_{\mathrm{x}}$. Resistive switching is well known to occur in $\mathrm{NiO}_{\mathrm{x}}$, supported by the presence of defects (e.g., oxygen vacancies). ${ }^{37}$ The behavior displayed by our $\mathrm{Ni} / \mathrm{NiO}_{\mathrm{x}}$ ! F8BT devices suggests that conductive filaments of metallic nickel are formed in the $\mathrm{NiO}_{\mathrm{x}}$ native layer upon the application of the voltage bias, causing the spikes in current. A fraction of these conductive filaments is likely to persist during the whole sweep and to give rise to the hysteresis; note that the $\mathrm{NiO}_{\mathrm{x}}$ layer formed on our electrodes during air exposure is expected to be thin (a few nanometers), ${ }^{38}$ resulting in large electric fields across it at the voltages used in this study. Supporting this interpretation, graphene-passivated electrodes do not show any significant hysteresis, while presenting a much higher density of injected current [Fig. 3(b)], consistent with the current densities in the large area device [Fig. 2(b)].

In conclusion, we were able to fabricate clean interfaces based on nickel passivated with few-layer graphene, providing efficient charge injection into solution-processed organic semiconductor layers. Crucially, we were able to fabricate such layers as bottom electrodes with very low roughness, which enabled their employment in large area vertical metal-semiconductor-metal devices, up to two orders of magnitude larger than that of the previously reported organic devices, based on graphene-passivated cobalt. ${ }^{10}$ Our results provide a route to retain two main advantages offered by organic semiconductors, large area and solution processing in air, while also producing high quality ferromagnetic electrodes with clean interfaces, the latter being a necessary requisite for achieving high performance optoelectronic and, particularly, spintronic devices.

We acknowledge funding from EPSRC (Nos. EP/P005152/1 and EP/M005143/1). R.M. and K.N. acknowledge funding from the EPSRC Cambridge NanoDTC (Grant No. EP/G037221/1). J.A.-W. acknowledges the support of his Research Fellowship from the Royal Commission for the Exhibition of 1851 and the Royal Society Dorothy Hodgkin Research Fellowship. R.S.W. acknowledges support from a CAMS-UK fellowship. A.I.A. acknowledges support from Marie Skłodowska-Curie Action (645725). Chris Amey is acknowledged for taking the XPS measurements.

Data sets related to this publication are available from the Cambridge University data repository at https://doi.org/10.17863/ CAM.51196.

\section{REFERENCES}

${ }^{1}$ C. Wang, H. Dong, W. Hu, Y. Liu, and D. Zhu, Chem. Rev. 112, 2208 (2012).

${ }^{2}$ F. So and D. Kondakov, Adv. Mater. 22, 3762 (2010).

${ }^{3}$ E. Voroshazi, B. Verreet, A. Buri, R. Müller, D. Di Nuzzo, and P. Heremans, Org. Electron. 12, 736 (2011).

${ }^{4}$ J. S. Bunch, S. S. Verbridge, J. S. Alden, A. M. Van Der Zande, J. M. Parpia, H. G. Craighead, and P. L. McEuen, Nano Lett. 8, 2458 (2008).

${ }^{5}$ V. Berry, Carbon 62, 1 (2013)

${ }^{6}$ A. A. Sagade, A. I. Aria, S. Edge, P. Melgari, B. Gieseking, B. C. Bayer, J. C. Meyer, D. Bird, P. Brewer, and S. Hofmann, Npj 2D Mater. Appl. 1, 35 (2017).

${ }^{7}$ Y. S. Dedkov and M. Fonin, New J. Phys. 12, 125004 (2010).

${ }^{8}$ M. B. Martin, B. Dlubak, R. S. Weatherup, M. Piquemal-Banci, H. Yang, R. Blume, R. Schloegl, S. Collin, F. Petroff, S. Hofmann, J. Robertson, A. Anane, A. Fert, and P. Seneor, Appl. Phys. Lett. 107, 012408 (2015).

${ }^{9}$ T. Verduci, C. S. Yang, L. Bernard, G. Lee, S. Boukari, E. Orgiu, P. Samorì, J. O. Lee, and B. Doudin, Adv. Mater. Interfaces 3, 1500501 (2016). 
${ }^{10}$ G. Zhou, G. Tang, T. Li, G. Pan, Z. Deng, and F. Zhang, J. Phys. D 50, 095001 (2017).

"A. Dahal and M. Batzill, Nanoscale 6, 2548 (2014).

${ }^{12}$ M. Piquemal-Banci, R. Galceran, M. B. Martin, F. Godel, A. Anane, F. Petroff, B. Dlubak, and P. Seneor, J. Phys. D 50, 203002 (2017).

${ }^{13}$ P. U. Asshoff, J. L. Sambricio, A. P. Rooney, S. Slizovskiy, A. Mishchenko, A. M. Rakowski, E. W. Hill, A. K. Geim, S. J. Haigh, V. I. Fal'Ko, I. J. Vera-Marun, and I. V. Grigorieva, 2D Mater. 4, 031004 (2017).

${ }^{14}$ M. B. Martin, B. Dlubak, R. S. Weatherup, H. Yang, C. Deranlot, K. Bouzehouane, F. Petroff, A. Anane, S. Hofmann, J. Robertson, A. Fert, and P. Seneor, ACS Nano 8, 7890 (2014).

${ }^{15}$ J. Devkota, R. Geng, R. C. Subedi, and T. D. Nguyen, Adv. Funct. Mater. 26, 3881 (2016)

${ }^{16}$ J. P. Prieto-Ruiz, S. G. Miralles, H. Prima-García, A. López-Muñoz, A. Riminucci, P. Graziosi, M. Aeschlimann, M. Cinchetti, V. A. Dediu, and E. Coronado, Adv. Mater. 31, 1806817 (2019).

${ }^{17}$ X. Sun, S. Vélez, A. Atxabal, A. Bedoya-Pinto, S. Parui, X. Zhu, R. Llopis, F. Casanova, and L. E. Hueso, Science 357, 677 (2017).

${ }^{18}$ D. Sun, E. Ehrenfreund, and Z. V. Vardeny, Chem. Commun. 50, 1781 (2014).

${ }^{19}$ S. Zanettini, G. Chaumy, P. Chávez, N. Leclerc, C. Etrillard, B. Leconte, F. Chevrier, J. F. Dayen, and B. Doudin, J. Phys. 27, 462001 (2015).

${ }^{20}$ H. Ma, H. L. Yip, F. Huang, and A. K. Y. Jen, Adv. Funct. Mater. 20, 1371 (2010).

${ }^{21}$ L. S. Liao, L. S. Hung, W. C. Chan, X. M. Ding, T. K. Sham, I. Bello, C. S. Lee, and S. T. Lee, Appl. Phys. Lett. 75, 1619 (1999).

${ }^{22}$ E. Ozceri and Y. Selamet, J. Phys. D 48, 455302 (2015).

${ }^{23}$ R. S. Weatherup, B. Dlubak, and S. Hofmann, ACS Nano 6, 9996 (2012).
${ }^{24}$ A. Cabrero-Vilatela, R. S. Weatherup, P. Braeuninger-Weimer, S. Caneva, and S. Hofmann, Nanoscale 8, 2149 (2016).

${ }^{25}$ A. C. Ferrari and D. M. Basko, Nat. Nanotechnol. 8, 235 (2013).

${ }^{26}$ R. S. Weatherup, L. D’Arsié, A. Cabrero-Vilatela, S. Caneva, R. Blume, J. Robertson, R. Schloegl, and S. Hofmann, J. Am. Chem. Soc. 137, 14358 (2015).

${ }^{27}$ B. L. Zink, M. Manno, L. O’Brien, J. Lotze, M. Weiler, D. Bassett, S. J. Mason, S. T. B. Goennenwein, M. Johnson, and C. Leighton, Phys. Rev. B 93, 184401 (2016).

${ }^{28}$ Y. Zhang and P. W. M. Blom, Appl. Phys. Lett. 98, 143504 (2011).

${ }^{29}$ M. C. Gwinner, R. D. Pietro, Y. Vaynzof, K. J. Greenberg, P. K. H. Ho, R. H. Friend, and H. Sirringhaus, Adv. Funct. Mater. 21, 1432 (2011).

${ }^{30}$ D. Kabra, L. P. Lu, M. H. Song, H. J. Snaith, and R. H. Friend, Adv. Mater. 22, 3194 (2010).

${ }^{31}$ H. Hibino, H. Kageshima, M. Kotsugi, F. Maeda, F. Z. Guo, and Y. Watanabe, Phys. Rev. B 79, 125437 (2009).

${ }^{32}$ S. M. Song, J. K. Park, O. J. Sul, and B. J. Cho, Nano Lett. 12, 3887 (2012).

${ }^{33}$ S. M. Song, J. H. Bong, and B. J. Cho, Appl. Phys. Lett. 104, 083512 (2014).

${ }^{34}$ A. M. Nardes, M. Kemerink, M. M. de Kok, E. Vinken, K. Maturova, and R. A. J. Janssen, Org. Electron. 9, 727 (2008).

${ }^{35}$ T. Ohta, A. Bostwick, T. Seyller, K. Horn, and E. Rotenberg, Science 313, 951 (2006).

${ }^{36}$ E. V. Castro, K. S. Novoselov, S. V. Morozov, N. M. R. Peres, J. M. B. L. Dos Santos, J. Nilsson, F. Guinea, A. K. Geim, and A. H. C. Neto, Phys. Rev. Lett. 99, 216802 (2007).

${ }^{37}$ H. D. Lee, B. Magyari-Köpe, and Y. Nishi, Phys. Rev. B 81, 193202 (2010).

${ }^{38}$ P. H. Holloway, J. Vac. Sci. Technol. 18, 653 (1981). 